\title{
BMJ Open MiYoga: a randomised controlled trial of a mindfulness movement programme based on hatha yoga principles for children with cerebral palsy: a study protocol
}

Catherine Mak, ${ }^{1,2}$ Koa Whittingham, ${ }^{1,2}$ Ross Cunnington, ${ }^{2}$ Roslyn N Boyd ${ }^{1}$

To cite: Mak C, Whittingham $\mathrm{K}$, Cunnington R, et al. MiYoga: a randomised controlled tria of a mindfulness movement programme based on hatha yoga principles for children with cerebral palsy: a study protocol. BMJ Open 2017;7:e015191. doi:10.1136/ bmjopen-2016-015191

- Prepublication history and additional material are available. To view these files, please visit the journal online (http://dx.doi. org/10.1136/bmjopen-2016015191).

Received 17 November 2016 Revised 27 March 2017 Accepted 18 May 2017

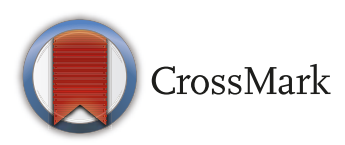

${ }^{1}$ Queensland Cerebral Palsy and Rehabilitation Research Centre (QCPRRC), The University of Queensland Child Health Research Centre (UQ-CHRC), Faculty of Medicine, The University of Queensland, Brisbane, Queensland, Australia

${ }^{2}$ School of Psychology, The University of Queensland, Brisbane, Queensland, Australia

Correspondence to

Catherine Mak;

c.mak@uq.edu.au

\section{ABSTRACT}

Introduction Cerebral palsy (CP) is the most common childhood physical disability, with life-long impacts for 1.77 in 1000 children. Although CP is primarily a physical disability, children with $\mathrm{CP}$ have an increased risk of experiencing cognitive difficulties, particularly attention and executive function deficits. Impairment in cognitive abilities can lead to subsequent impairment in independent functioning, education, employment and interpersonal relationships. This paper reports the protocol of a randomised controlled trial of a novel family-centred lifestyle intervention based on mindfulness and hatha yoga principles (MiYoga). MiYoga aims to enhance child and parent outcomes for children with CP.

Methods and analysis The aim is to recruit 36 childparent dyads (children aged 6-16 years; bilateral or unilateral CP; Gross Motor Function Classification System I-III), who will be randomly assigned to two groups: MiYoga and wait list control. The MiYoga programme will be facilitated in a group format for 8 weeks. Assessments will be administered at baseline, prior to MiYoga, following completion of MiYoga, and at 6-month followup (retention). The primary outcome will be the child's sustained attentional ability as measured by the Conner's Continuous Performance Test II. Other outcomes of interest for children with $\mathrm{CP}$ consists of attentional control, physical functioning, behavioural and well-being. For parents, the outcomes of interest are mindfulness, psychological flexibility and well-being. Data will be analysed using general linear models, specifically analysis of covariance and analysis of variance.

Ethics and dissemination Full ethical approval for this study has been obtained by the Children's Health Queensland Hospital and Health Service Research Ethics Committee (HREC/12/QRCH/120) and The University of Queensland (2012000993). If MiYoga is proven effective, its dissemination would assist children with $\mathrm{CP}$ and complement their ongoing therapy by improving the ability of the child to pay attention at school and in therapy, and alleviating environmental stressors for both the child and his/her parents.

Trial registration number ACTRN12613000729729;

Pre-results.http://www.ANZCTR.org.au/

ACTRN12613000729729.aspx
Strengths and limitations of this study

- To our knowledge, this is the first randomised controlled trial (RCT) exploring the benefits of a mindfulness-based movement intervention for children with cerebral palsy.

- Mindfulness and hatha yoga (MiYoga) is designed to be a lifestyle intervention so that children and parents will be able to continue practice at home in their everyday lives after the completion of the programme.

- MiYoga is fun and developmentally appropriate and is adaptable to the needs of the child and/or parent.

- All participants will be offered MiYoga before completion of the study for ethical reasons.

- Participants in this study span across a broad age range (aged 6-16 years) and within which there maybe variability in cognition and executive function. Executive function is a secondary measure in the study.

- There is a high number of secondary outcomes in this study, which would be exploratory in nature as this is the first RCT to investigate the effects of mindfulness and yoga in children with cerebral palsy.

Date of trial registration Prospectively registered on 2 July 2013-present (ongoing).

Findings to date Recruitment is complete. Data are still being collected at present. We aim to complete data collection by February 2017.

\section{INTRODUCTION}

Cerebral palsy (CP) is the most common physical disability in childhood with lifelong impacts for 1.77 in 1000 children. $^{1}$ $\mathrm{CP}$ is caused by non-progressive damage to the fetal or infant brain that results in motor and postural difficulties. ${ }^{2}$ In addition to physical impairments, which are the diagnostic features of CP, children can also experience cognitive and psychological difficulties. ${ }^{3-5}$ 
The motor and cognitive difficulties are often accompanied by disturbances of sensation, communication, perception, behaviour problems and/or by a seizure disorder. $^{24}$

Present therapies focus primarily on physical difficulties faced by children with $\mathrm{CP}^{6}{ }^{6}$ despite the fact that children with $\mathrm{CP}$ also have an increased risk of experiencing cognitive difficulties. In 2009, the Australia Cerebral Palsy Register reported that one in three children with $\mathrm{CP}$ experience cognitive difficulties. ${ }^{7}$ More recent studies reported that one in two children with $\mathrm{CP}$ had an intellectual disability, ${ }^{4}$ and compared with typically developing children those with CP are more likely to have attention and executive function deficits. ${ }^{8-10}$ The deficits may explain why children with CP have increased social and learning problems. ${ }^{11}$ It is therefore important to consider interventions that target attention, in addition to the existing physical therapy, for children with CP.

\section{Attention}

The attention system underpins most cognitive and neuropsychological functions in everyday life, such as self-control (inhibition), decision-making, goal setting, planning, problem solving, emotional responses and behaviour. Typically, the attention system is understood using the 'three network model', comprising the alerting network, and the orienting and executive networks. ${ }^{12}$

The alerting network is a 'bottom-up', stimulus-driven (or exogenous) attention system that automatically responds to external stimulus, such as visual, auditory input. ${ }^{13}$ For example, a sudden loud noise or motion can attract our attention and we attend to them whether we want to or not. This network is thought to involve both the parietal and temporal areas of the brain, as well as the brain stem arousal systems. ${ }^{12}$

Orienting and executive networks are the other networks in this model. In contrast to the alerting network, they are both 'top-down' processes, responding to internally determined goals. Together, these two attentional networks are also known as goal-driven, endogenous attention, attentional control or executive attention networks. ${ }^{12}$ They determine our ability to voluntarily select, and orient, attention to relevant stimuli or tasks, mediated primarily by the frontal cortex and basal ganglia. ${ }^{12} 14$

The orienting network determines our ability to consciously orient attention in a spatial setting. Functionality of this network can be measured by the speed and accuracy of responses to cues that indicate where a future stimulus may occur on a computer screen.

In contrast, the executive network involves mechanisms for monitoring and resolving conflict when two responses are simultaneously called for by an external stimulus. This network aids attentional switching, and provides the ability to disengage from distracting stimuli and re-engage attention to the relevant task or stimulus. Previous studies have confirmed that these two 'top-down' networks are related to executive functions, ${ }^{12}{ }^{15}$ working memory ${ }^{16}$ and conflict resolution and inhibition. ${ }^{17}$
Both orienting and executive networks of attention are involved in sustained attention, which is the ability to maintain and focus attention on a given task. Sustained attention is a core executive function, typically defined as the ability to maintain an alert, goal-directed focus in the absence of an external stimulation. ${ }^{18}$ Difficulty maintaining sustained attention can interfere with non-cognitive aspects of therapy and rehabilitation, including for children with CP. Impairments in attention in children may lead to reading problems, ${ }^{19}$ poor math achievements, ${ }^{20}{ }^{21}$ poor self-confidence and cognitive impairments, ${ }^{22}$ poor executive function and executive attention (such as working memory) leading to poor social skills ${ }^{11} 23$ as well as problem behaviours. ${ }^{11}$ Furthermore, the capacity to self-sustain attention has been shown to predict motor recovery in adults following a right hemisphere stroke over a 2-year period. ${ }^{24}$ These findings suggests sustained attention deficits in the development of interventions and rehabilitation programmes are a worthwhile target for interventions, particularly in children with $\mathrm{CP}$, where poor attention may hinder their ability to benefit from existing physical therapies.

\section{Cognitive abilities in children with CP}

Attention, executive function and other intellectual capabilities are impaired in one in two children with CP. ${ }^{348925}$ One cross-sectional study of sustained attention in 10 adolescents with CP, using a visual Continuous Performance Test (CPT), ${ }^{26}$ indicated that sustained attention ability was affected by $\mathrm{CP}$, even after controlling for motor deficits by using eye movements to measure sustained attention. ${ }^{9}$ Another study investigated attention and executive function in children with unilateral and bilateral spastic CP. ${ }^{8}$ Four subtests from the Wechsler Intelligence Scale for Children (WISC-III) ${ }^{27}$-information, similarities, comprehension and vocabulary-were used to measure general cognitive functioning. They also used three subtests from the Test of Everyday Attention for Children $^{28}$ to measure selective attention (Sky Search), sustained attention (Score!) and divided attention (Sky Search Dual Task). Executive functions were assessed by the Contingency Naming Test and the teacher version of the Behaviour Rating Inventory of Executive Function (BRIEF). ${ }^{29}$ The results demonstrated that impairments in sustained attention, divided attention, inhibition, switching and general executive function were present in children with CP.

Few therapeutic interventions aim to increase a child's ability to sustain their attention on task, or selectively focus their attention on appropriate stimuli. Previous research has explored attention and problem-solving training for children with attention deficit hyperactivity disorder (ADHD),${ }^{30}$ children with learning disabilities and comorbid ADHD. ${ }^{31}$ In addition, some studies investigated efficacy of interventions that may increase attentional abilities in children with traumatic brain injury ${ }^{30} 32$ and for the late effects of treatment for childhood cancer. ${ }^{33}$ 
There is a paucity of research on interventions to improve attention in children with CP.

\section{Mindfulness and attention}

Several investigators have recently proposed theoretical explanations of how the practice of focused attention through mindfulness (awareness of the present moment) training can enhance and develop attention regulation. ${ }^{3435}$ Mindfulness practice involves sustaining attention on a chosen object, such as one's breath, an object or a mantra, and directing attention to that object while sustaining this focused attention for as long as possible. ${ }^{34-38}$ In order to sustain this focus, one must monitor the quality of this focus. For example, while focusing on the rise and fall of the chest with each breath, one might notice their attention has shifted to something that happened during the day, or what they would like for dinner later. At some point, they would realise their focus has wondered and let go of their distraction and reattend to their breath. Mindfulness is the state of awareness of the thoughts or feelings that arise when our minds wander. This awareness provides the opportunity to inhibit the more dominant response of rumination and switch our attention back to the point of focus.

It has been proposed ${ }^{35}$ that this type of monitoring of one's attention and focus consists of three types of attention regulation: (i) monitoring the present moment (ie, detect mind wandering), (ii) attentional switching (ie, disengage from a distracting object/thought without further involvement) and (iii) selective attention (ie, ability to redirect focus promptly back to target object). Researchers reason that mindfulness training entails extended practice of these attentional abilities. ${ }^{3435} 37$

These proposed theoretical models of the cognitive mechanism of mindfulness have inspired experimental research examining the effects of mindfulness training on attention performance. The effects of mindfulness practice on the three attentional networks (alerting, orienting and executive) were examined by Jha $e t a l^{\beta \gamma}$ using the Attention Network Test. ${ }^{39}$ Attentional efficiency was assessed before and after an 8-week mindfulness-based stress reduction (MBSR $)^{40}$ course administered to meditation-naïve participants, as well as a 1-month intensive mindfulness retreat attended by experienced meditators, compared with an 8-week no treatment control group. The retreat group (experienced meditators) showed better conflict monitoring at baseline than participants in the control and MBSR groups (both groups with meditation-naïve participants), suggesting that executive attention improves with long-term exposure to mindfulness meditation. The retreat group had improved alerting among the previously experienced meditators. The authors concluded that mindfulness training improves voluntary (top-down; endogenous) attentional control leading to improved orienting and conflict monitoring, and prior experience with concentrative meditation allows for the development of improved bottom-up, receptive attention involved in alerting. It may be that mindfulness training improves the functioning of each of the attentional subsystems at various points in the course of mindfulness training.

\section{Yoga}

Traditional yoga is a mindfulness practice that directs attention towards the present moment. ${ }^{41}$ The aim of yoga is to calm and unify the mind, body and breath to promote positive health, self-awareness and spirituality. ${ }^{42}$ Yoga postures, known as asana in Sanskrit, are the third of the eight 'limbs', or practices, of yoga according to the traditional texts of the YogaSūtrass of Patañjali. Asana directly translates to 'posture' and was derived from the Sanskrit root word which means 'to be', 'to sit', 'to stay' or 'to be established in a particular posture'. ${ }^{43}$ The YogaSūtrass of Patañjali describe asana as having two important qualities. The first is steadiness and alertness and the second is the ability to remain comfortable in a posture. ${ }^{42}$ When practicing asana, both of these qualities should be present. The combination of performing asana with pranayama (breathing techniques), comprise the style of yoga commonly referred to as Hatha Yoga. Hatha Yoga unites the actions of the body, breath and mind by the synchronisation of movement with the breath, which in turn directs the practitioner's attention and awareness to the present. The unity of body and mind is what makes traditional Hatha Yoga an embodied mindfulness practice.

\section{Mindfulness and yoga for children}

In the paediatric literature, it has been documented that mindfulness and yoga improves memory in school children, ${ }^{44}$ cognitive function in children with mental retardation, ${ }^{45}$ planning abilities and executive function in healthy school aged girls ${ }^{46}$ as well as attention, behaviour and emotional control in healthy children and children with ADHD. ${ }^{47}$ Yoga has been shown to improve balance, motors skills and strength as well as the quality of life and general well-being, in a range of different participant groups. ${ }^{48-51}$

Mindfulness-based stress reduction $^{52}$ programmes combine mindfulness and Hatha Yoga techniques to cultivate greater awareness of the unity of mind and body, as well as of the ways the unconscious thoughts, feelings and behaviours can undermine emotional, physical and spiritual health. The underlying principles for MBSR programmes are to (a) be non-judgmental, (b) be patient, (c) see the world as if for the first time, (d) accept seeing things as they are without trying to change anything, (e) and let go of attachment to repetitive thoughts, actions or beliefs. MBSR programmes usually feature group interaction, formal and informal meditation practice, daily homework and/or silent retreats. ${ }^{52}$ The formal practice includes mindfulness meditation and mindful movements in the form of Hatha Yoga. The informal practices include mindful eating and walking. Although a number of studies explored the effectiveness of MBSR in the adult population, ${ }^{53} 54$ there is a paucity 
of randomised controlled trials that explore the effects of a developmentally appropriate MBSR programme for children.

A systematic review ${ }^{55}$ investigating the efficacy of mindfulness-based interventions identified 13 randomised controlled trials that met the inclusion criteria. Out of the 13 identified studies, ${ }^{56-68} 5$ studies found a statistically significant intervention effect for at least one outcome measure of attention or executive function with medium to large effects sizes $(0.3-32.03) .{ }^{5759616366}$ The overall findings from the review suggested that mindfulness-based interventions targeting attention are a promising approach for children and adolescents. This systematic review did not find randomised controlled trial of mindfulness-based interventions with children or adolescents with CP. Quality trials are therefore needed to assess the effectiveness of mindfulness-based interventions targeting attention in children and adolescents with CP.

By adapting a mindfulness-base programme for children, with a focus on mindful movements through Hatha Yoga, it may provide a developmental approach to mindfulness training. Yoga has been shown to improve body awareness in adults, ${ }^{69}$ and it may teach children and adolescents about their growing bodies as they become more aware of their bodies and their movements. School-age children have also been found to be interested in yoga as a mind-body therapy for pain and other ailments. ${ }^{70}$ As children are naturally more active, teaching them mindfulness through movements using traditional Hatha Yoga postures along with the some mindfulness exercises to begin with, may be more developmentally appropriate than teaching them the more traditional seated mindfulness meditations. This remains to be tested.

The mindful movement programme in this project, MiYoga, combines both cognitive (mindfulness) and physical (yoga postures) aspects into therapy. It has the potential to assist children with CP to alleviate negative effects of environmental stressors by improving their ability to focus attention on the present moment, ultimately helping them to focus on activities at school, in therapy, or when interacting with peers. At the same time, Hatha Yoga may provide additional physical benefits to complement the ongoing therapy for children with CP.

MiYoga will be delivered to both the children and their parents, to enable parents to facilitate and monitor the MiYoga approach, which may improve translation and implementation of techniques into everyday life. This project will test the efficacy of a mindful movement (MiYoga) programme administered to children with $\mathrm{CP}$, and one of their parents. The MiYoga programme would be an adjunct to children's ongoing rehabilitation therapy.

\section{Aim}

The focus of this study is to design and test a novel mindful movement programme, MiYoga, and to investigate its efficacy for enhancing sustained attention in children with CP. Efficacy will be tested in a randomised controlled trial $(n=36)$ with an intervention group (MiYoga programme+standard care) compared with a waitlist control group receiving standard care alone. This enables all participating children to receive the intervention, maximising participant recruitment and retention and fulfilling ethical obligations to participating children and families. Outcomes measures will be taken at baseline, immediately post-intervention and at follow-up 6 months post-intervention.

Qualitative interviews will also be conducted with each child and parent participant individually. The aim of these interviews is to collect the participants' perceptions of the MiYoga programme.

\section{Hypotheses}

It is hypothesised that, compared with the waitlist control group, the MiYoga group will show improvements in the following child outcomes:

1. sustained attention (Conner's CPT II) ${ }^{26}$;

2. executive function (information processing, attentional control, cognitive flexibility, working memory and behavioural manifestations of executive function in daily life);

3. psychological functioning (Strengths and Difficulties Questionnaire (SDQ) $)^{71}$;

4. physical capacity including functional strength (sit-tostand, half-kneel to stand and lateral step-up tests) ${ }^{72}$ and flexibility (sit and reach test);

5. present-moment awareness (Child and Adolescence Mindfulness Measure (CAMM) $)^{73}$;

6. quality of life (CP-QOL-Child or CP-QOL-Teen). ${ }^{74} 75$

In addition, it is hypothesised that the MiYoga group will show improvements in the following parent outcomes:

1. mindfulness (Mindfulness Awareness Attention Scale $($ MAAS $))^{76}$;

2. parent psychological well-being (Depression Anxiety and Stress Scale (DASS) $)^{77}$;

3. parent's psychological flexibility (Acceptance and Action Questionnaire ((AAQ-2) $)^{78}$;

4. parent's personal well-being (Personal Well-being Index-Adults (PWI-A) $)^{79}$;

5. child-parent relationship (Child-Parent Relationship Scale (CPRS) $).{ }^{80}$

\section{METHODS AND ANALYSIS \\ Ethics}

Full ethical approval for this study has been obtained by the Behavioral and Social Sciences Ethical Review Committee of The University of Queensland (2012000993) and Children's Health Queensland Hospital and Health Service Research Ethics Committee (HREC/12/QRCH/120). Protocol modifications and amendments will be submitted to the ethical committees for approval. This trial has been registered with the Australian New Zealand Clinical Trials 
Registry (ACTRN12613000729729). All families will be given written informed consent to participate and their parents or guardians prior to entering into the trial.

\section{Recruitment}

Thirty-six children with a diagnosis of $\mathrm{CP}$ with spastic motor type, either diplegia (bilateral CP) or hemiplegia (unilateral CP) motor distribution, and one of their parents, will be recruited into the MiYoga study. Children must also have sufficient cooperation and cognitive understanding to follow simple instructions. The primary parent of eligible children will participate alongside their child. Children with comorbid diagnoses to CP are included.

Children will be excluded if they have (i) received upperlimb or lower-limb surgery in the previous 6 months; (ii) unstable epilepsy (ie, frequent seizures not controlled by medication); (iii) spinal instability or other spinal problems that would prevent them from participating safely in the MiYoga programme; (iv) have a medical condition that would prevent them from participating safely in the MiYoga programme or (v) parents/guardians who are pregnant as a safety precaution. Diagnosis of CP must be confirmed by the treating paediatrician or clinician and in accordance with published recommendations.

Eligible children living in South East Queensland, Australia, will be recruited through patient databases of Queensland Cerebral Palsy Rehabilitation Research Centre (QCPRRC) at the Royal Children's Hospital and the Queensland Cerebral Palsy Register, and via referrals from clinicians in the Queensland Pediatric Rehabilitation Service. Potential participants will be invited to participate in the study via letters and follow-up phone calls. Flyers and posters will displayed in the reception areas of clinics, and clinicians will be informed of the study, and encouraged to refer appropriate patients to the programme. External advertising will include a webpage on The University of Queensland School of Psychology website and a study flyer or a brief description of the study will also be advertised in some South East Queensland schools' newsletters and notice boards.

Participants will be assessed for eligibility and enrolled in the study by the study coordinator. The study will be explained to parents by a member of the research team, and signed written consent will be obtained, acknowledging that the children and parents are aware of all study requirements, consent to take part, give permission for researchers to access their child's medical records, agree to the video-recording and photographs of sessions and understand that they are free to withdraw their consent at any time. It is anticipated that enrolment will commence in September 2013.

\section{Design}

This study will use a randomised controlled trial design to evaluate the efficacy of the MiYoga programme compared with waitlist control (care as usual) for children with CP, aged 6-16 years (see figure 1). After each child and parent completes their baseline assessments, they will be randomly allocated to one of two groups:

1. Immediate MiYoga group (group 1)—families commence MiYoga programme immediately for 8 weeks.

2. Waitlist MiYoga control (group 2)-families continue care as usual for 8 weeks and then are reassessed before commencing a future MiYoga programme. Care as usual consists of any therapies (eg, occupational therapy, physiotherapy, psychologist) usually provided by public or private services and visits to their treating paediatrician or general practitioner.

To limit confounding variables during the immediate intervention period (baseline to 8-week follow-up), children scheduled to receive casting, splinting or intramuscular botulinumum toxin type A (BoNT-A) injections during the immediate intervention period will not be eligible. Participants who have received intramuscular BoNT-A prior to beginning the study will have their baseline assessments postponed until 1 month after their standard follow-up has been completed. Questionnaires will record the type, frequency and duration of any concurrent therapies provided by public and private services during the study for all participants.

\section{Randomisation}

Prior to the intervention, participants will be asked to complete baseline assessments and questionnaires. Once these are completed, participants will be randomly assigned to either the waitlist group or immediate group and informed of the dates and times of their group. The randomisation sequence will be computer-generated. Treatment allocations will be recorded in pieces of paper, which will be folded and placed inside sealed, numbered, opaque envelopes by a staff member not involved in the study. A staff member not involved in the study will also open consecutive envelopes as each participant returns their baseline questionnaires. After the randomisation process is complete, study personnel will be informed of group allocation and information packs will be sent out to families. Given the nature of the study, no parties will be blinded to group assignment. The experimental design and outcome measures are depicted in figure 1.

\section{Adverse events}

There are no known health or safety risks associated with participation in the described study, and the risk of adverse events is low. The ethical review process and conduct of the trial is monitored by the two ethics committees, therefore, no additional Data Monitoring Committee is considered necessary.

Any minor or major events associated with intervention or usual care groups will be monitored throughout the 8-week programme duration. The chief investigators KW and RB will review any adverse events or unintended effect detected. 


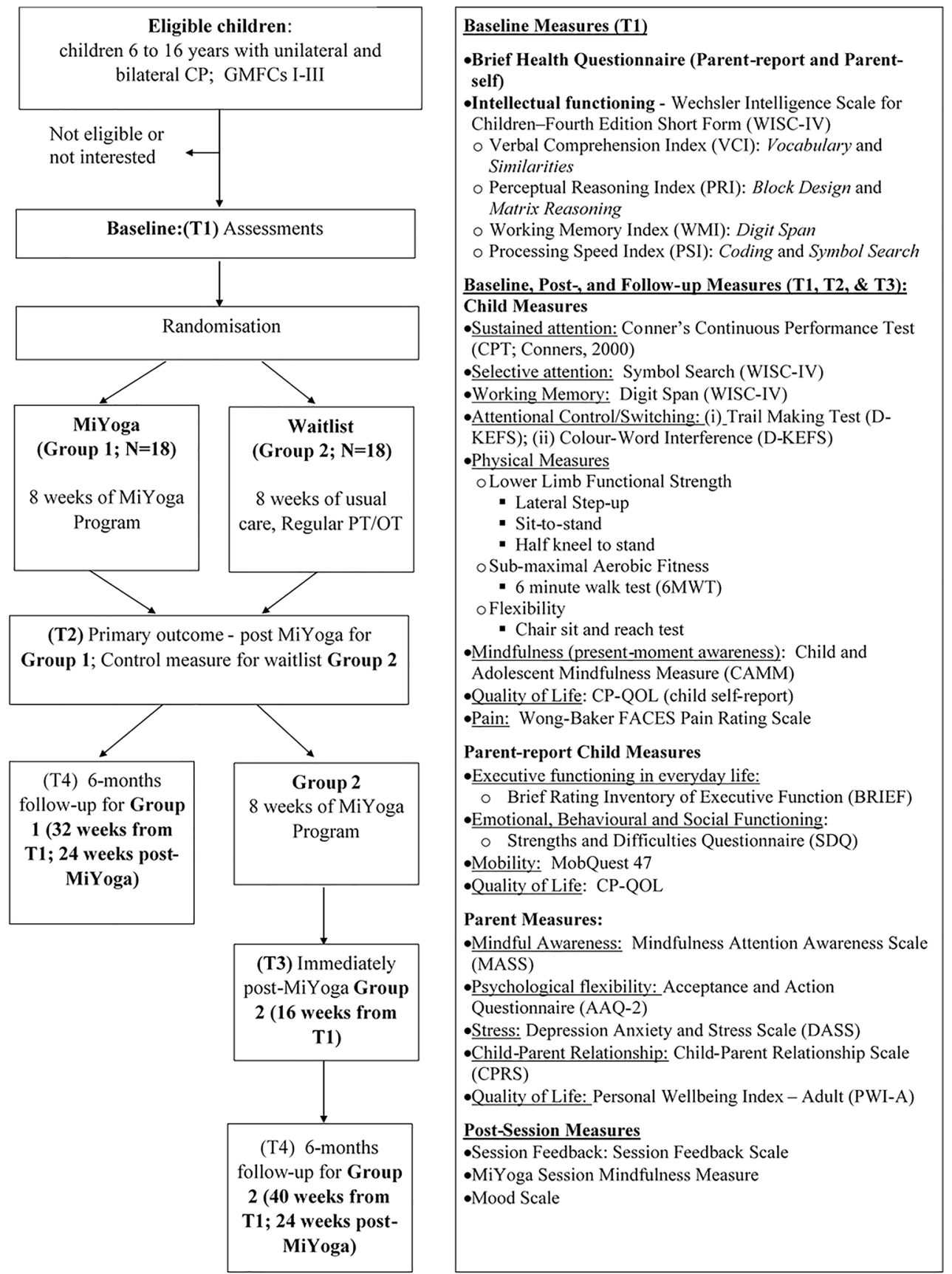

Figure 1 Flow chart of MiYoga study design.

\section{Study procedure}

All participants will attend baseline assessments (T1) before being randomly allocated into either the immediate MiYoga group or the waitlist control group. Participants in the immediate group will begin the 8-week MiYoga programme within 3 weeks of their baseline assessment, while the waitlist group will continue care as usual for the next 8 weeks. When the immediate group has completed the MiYoga programme, all participants from both groups (immediate and waitlist) will be reassessed (T2). The waitlist group can then commence a future MiYoga group of their choice. For each participant, data will be collected at baseline (T1) and following the immediate MiYoga group's completion of

the programme at 8 weeks (T2). The waitlist group will also complete assessments after they have completed the MiYoga programme at 16 weeks (T3). Both groups will complete their follow-up assessments 24 weeks after completing the MiYoga programme-this will be 32 weeks post-baseline for the immediate group and 40 weeks post-baseline for the waitlist group (T4). Participants will complete their assessments within 3 weeks of the target time-points (in weeks) listed above.

The principal researcher (CM), a registered psychologist and a qualified yoga teacher, will conduct all the assessments and MiYoga sessions. A physiotherapist from the QCPRRC will perform the physical screen assessment for each child at baseline. In addition, they will be present 
at the initial assessment to provide professional advice to the child, the parent/guardian and to the psychologist/ yoga teacher regarding the child's ability to perform various yoga postures and the adaptations that may be necessary. Either a physiotherapist or the principal researcher (CM) will conduct the physical assessments. Participants are encouraged to complete their online questionnaires in person during their assessment session, if they prefer they may also complete them online from home (via Qualtrics Insight Platform). Registered clinical and developmental psychologist (KW) will provide regular supervision. In addition, study coordinator will be able to consult with Professor Boyd (RB), a registered physiotherapist, if there are any physical concerns.

\section{MiYoga intervention}

Following randomisation (immediate MiYoga group), or the time 2 neuropsychological and physical assessments (waitlist control group), participants and their parents will complete the MiYoga programme. All MiYoga sessions will be delivered in a group format where possible. Child-parent dyads in the waitlist control group will receive the same intervention after a delay. In two-parent families, one of the parents will be invited to attend all sessions, and the same parent will complete all the questionnaire measures.

The MiYoga programme itself is an 8-week programme that consists of six 90 min sessions over the initial 6 weeks, then two phone or Skype consultations over the last 2 weeks of the programme. Over these 8 weeks, children and their parent or guardian are asked to partake in a minimum of $20 \mathrm{~min}$ daily home practice. To assist with participants' home practice, a MiYoga DVD and a MiYoga poster developed by the principal researcher will be provided at the first MiYoga session. The MiYoga DVD is based on the mindfulness exercises and asana delivered in the group sessions. The MiYoga poster shows pictorially the asana delivered in the group sessions. In addition, it includes child specific modifications identified from the initial assessment session. Each MiYoga poster will contain general instructions as well as general and specific modifications for how parents may assist their child. Where possible, a photo of the child performing the postures with their modifications will also be included. Researchers will assess the child's abilities in each of the yoga postures and provide modifications for the child as required in each posture. These modifications will be documented and put into the respective child's poster for them to take home and practice. This will ensure that participants will not attempt any new exercises or poses at home without assistance and guidance from a therapist (see online supplementary appendix A for a MiYoga programme summary).

There are formal and informal mindfulness activities in MiYoga. The formal mindfulness activities consist of mindful movements in the form of Hatha Yoga and short mindfulness meditations. The yoga postures selected for the programme aim to help them build strength and increase flexibility (see online supplementary appendix B for a table and illustrations of the MiYoga postures for children with cerebral palsy). The informal activities present mindfulness techniques as game-like explorations, based on exploring our internal and external worlds with the five senses as well as consciously reflecting on thoughts and feelings. These activities and games were developed based on literature on mindfulness and yoga for both children and adults. ${ }^{42} 435281-91$

One of the informal activities involves children having to guess what they are eating while blindfolded. This game will involve the child to consciously notice the food with all their senses, such as by feeling the texture of the food in their hands and mouth, smelling and tasting it and possibly even hearing the sounds the food makes when chewing it (and subsequently noticing crunchy, chewy or soft foods). Children may have to identify an object by exploring it only with their hands (sense of touch) and then describe how the object feels in their hands to another person without naming the object. This game encourages the child to use their sense of touch to explore their environment and increase their awareness of how the sense of touch provides them with information about their surroundings. Such activities are both interesting for the participants, but are also mindful practices that stimulate a state of awareness.

The Hatha Yoga techniques or postures in this study were based on Viniyoga principles ${ }^{39}$ and they were tailored for children with CP. The synchronisation of breath and movement is a key element as it helps to focus the mind on the present (ie, moving through yoga postures with the breath) providing a mindful movement practice. To make synchronising breath and movement more natural and fun for children, participants will be invited to make sounds along with the instructor, such as 'Ahh', in the asana sequences to assist with their exhalation. The yoga postures will be modified as required for each participant. Some of the modifications will use supports such as a wall, chair or blocks, to ensure correct posture, adequate support and comfort, or to accommodate muscle shortness or postural difficulties. For example, some children may sit on a block to keep their pelvis level and to accommodate their shorter hamstring muscles and leading to posture pelvic tilt within long sitting. These physical modifications will be individualised to each child's need based on the baseline physical screen by a physiotherapist. The physical adaptations may not be the same for all children with CP while some children did not require any physical modifications. For this reason, online supplementary appendix B mainly illustrates non-adapted postures and only some examples of possible modifications. In addition to the physical modifications, verbal prompts will guide the children into the postures. For example, 'going as far as it is comfortable for you, this may be coming forward to thighs or your knees' or 'notice the sensation in the back of your legs as you fold forward, you may like to explore how it feels for you to keep your knees straight as you come forward'. 
A verbal prompt for children to sit with good alignment (avoid W-sitting) may be 'as we sit back onto our heels be mindful that your knees and big toes are together'.

Each session will be of 90 min with guided instruction throughout each component of mindfulness practice. During Hatha Yoga postures, instructions will be provided on safe ways to get in and out of the positions, pose modification and use of props to suit needs and limitations of each child. For instance, the supine twist can be performed on a chair rather than on the ground. Blankets, cushions, bolsters, straps and other props may be used to maintain body alignment, structural support and comfort (see the possible modification column of online supplementary appendix B for more details). Emphasis will be placed on building length along the spine while maintaining neutrality of spinal position, keeping awareness of the breath and using breath and sensations within the body to anchor attention to the present moment.

\section{Locations}

It is expected that groups and assessments will be conducted in Brisbane, and various locations in South East Queensland. Locations in Brisbane will include The University of Queensland, St Lucia and Herston campuses and the Centre for Children's Health Research in South Brisbane. If participants are located outside of Brisbane, then suitable locations will be organised with the participating families in those areas.

\section{Participation and data management}

To maximise participation and retention, email reminders will be sent to participants 3 days prior to their scheduled appointments, and text message reminders will be sent to participants the day prior to their appointments and their weekly MiYoga sessions. To further promote recruitment, participation and retention, the study offers waitlist control group so that all participants have an opportunity to take part in the MiYoga programme. It is also possible to extend the period of recruitment and the delivery MiYoga groups should recruitment be more challenging than anticipated. The number of participants recruited and participant retention will be recorded throughout the trial period.

Treatment dose will be calculated based on participants' attendance at the weekly MiYoga sessions, as well as the amount of home practice completed by participants between sessions. The therapist will record participant attendance after each weekly MiYoga session, and parent participants will record the amount of home practice they completed each week in their MiYoga diary. The MiYoga diaries will be collected weekly and monitored by the therapist. Strategies to facilitate and support engagement in the programme will be discussed with the participant and their parents throughout the duration of the MiYoga programme in the group sessions as well as via individual phone calls.

A protocol checklist will be completed by the therapist (first author) at the end of each MiYoga session. This protocol checklist will be used to monitor therapy fidelity and to ensure that all the MiYoga content was delivered as per protocol.

Paper copy data will be de-identified and physically stored in a locked file cabinet at the QCPRRC. These physical documents may be scanned (after de-identification) and password protected in a computer file on a secure server. Computer files will be de-identified and password protected on a secure server.

Video files of the MiYoga sessions will be used to assess for therapist integrity and consistency across groups. These video files will be managed and kept on a secure online database run through QCPRRC and backup files may be kept on an external harddrive or CD in a locked cabinet.

Study updates and general outcomes will be sent to participants in a newsletter format. A summary report of the overall study findings will also be sent to participants at the conclusion of the project. Feedback on the specific formal assessments will be provided when requested by the participant or parent.

\section{Participant characteristic measures}

The following questionnaires and assessments will be completed prior to the intervention to gather baseline information about the sample of children and their parents:

\section{Gross Motor Function Classification System ${ }^{92}$}

The Gross Motor Function Classification System (GMFCS) classifies, across five levels, a child's ability to carry out self-initiated sitting and walking movements. ${ }^{92}$ A correlation between the GMFCS and the Gross Motor Function Measure (GMFM) of $\mathrm{r}=0.91$ suggests construct validity has been obtained. ${ }^{93}$ GMFCS also has good inter-observer reliability between professionals as well as between professionals and parents in children with CP. ${ }^{94}$

\section{Brief Health Questionnaire}

The Brief Health Questionnaire screens for existing medical conditions that may prevent the child or the parent to participant in MiYoga, with separate questionnaires for children and parents (see online supplementary appendix $\mathrm{C}$ ).

\section{Physical screening clinical examination}

A physiotherapist will conduct this examination to determine if the participating child has any physical conditions that might preclude them from exercise (see online supplementary appendix D).

WISC for Children—fourth edition short form (Wechsler, 2003) ${ }^{95}$

Intellectual functioning will be assessed using the WISCfourth edition (WISC-IV) short form. ${ }^{95}$ The WISC-IV comprises seven subtests that generate the four indices of Verbal Comprehension (VCI), Perceptual Reasoning (PRI), Working Memory (WM) and Processing Speed (PSI). From these overall short form index scores, a Full-Scale Intelligence Quotient (FSIQ) score can be calculated. In the WISC-IV short form, the VCI index is 
made up of the Vocabulary and Similarities subtests. The Vocabulary subtest assesses knowledge of word meanings and will require participants to name pictures or provide spoken definitions of words (eg, 'what is a bicycle?'). The Similarities subtest assesses verbal abstraction and reasoning and will require participants to describe how two words are similar (eg, 'how are anger and joy alike'). The Block Design and Matrix Reasoning subtests make up the PRI index in the WISC-IV short form. Block Design will require participants to construct abstract visual designs with a set of red-and-white 3D blocks within a specified time period. Matrix Reasoning, will require participants to use their non-verbal abstract reasoning and problem solving skills in order to select the correct picture from an array of five options that fits into the missing space of a visual design. The WMI index is made up of the Digit Span subtest in which participants will be required to repeat a number of verbally presented digits in forward and reverse order. Finally, the PRI index is made up of the Coding and Symbol Search subtests, each of which has a $2 \mathrm{~min}$ time limit and therefore require participants to work as quickly as possible without making mistakes. For the Coding subtest, participants will need to match abstract symbols with a number key while for Symbol Search, participants will be required to detect the presence of one or more symbols in a sequence of five. ${ }^{96}$

Scaled scores will be derived from index scores in accordance with normative data based on the child's age and gender $\left(\right.$ mean=100, SD=15) ${ }^{95} 97$ All index scores of the WISV-IV SF have shown moderate-to-high levels of internal consistency $(\alpha=0.87-0.96)$ and are equivalent to those documented for the full WISV-IV, with the exception of the WMI which is marginally lower than its full-length equivalent.

\section{Primary outcome measures \\ Attention}

The Conner's CPT II $^{26}$ will be used to measure sustained attention performance. It is a standardised $14 \mathrm{~min}$ computer administered test in which participants are asked to observe letters displayed on a computer screen and click at the appearance of each letter, except for that of the letter ' $\mathrm{X}$ '. The stimuli appear at random intervals (1, 2 or $4 \mathrm{~s})$ and the duration of each stimulus is $250 \mathrm{~ms}$. This test measures the time to process information (eg, reaction time (RT)) and errors such as false negatives or false positives. The test is divided into six equal blocks of 20 trials.

Twelve indices from the Conner's CPT will be analysed. These indices are: (i) number of Omission errors: the number of letters, other than X, not detected (testretest correlation coefficients $\mathrm{r}=0.84$ ); (ii) false positives/ Commission errors: the number of responses to the letter $\mathrm{X}(\mathrm{r}=0.65)$; (iii) Hit RT ( $\mathrm{r}=0.55)$; (iv) Hit RT SE ( $\mathrm{r}=0.65)$ and (v) Variability of RT: indicates consistency of RTs between blocks ( $\mathrm{r}=0.60)$; vi) Detectability ( $d$ prime): RT distribution of target versus non-target (X) $(\mathrm{r}=0.76)$; (vii) Perseverations: responses with a RT too short (5100 $\mathrm{ms}$ ) indicating anticipatory responding or responses to a previous stimuli (suggesting very slow responses/inattentiveness) ( $\mathrm{r}=0.43$ ); (viii) Response Style: indicates an overcautious versus a highly impulsive response $(\mathrm{r}=0.62)$; (ix) Hit RT Block Change: a high score implies that, as the test progresses, RTs are longer $(\mathrm{r}=0.28)$; $(\mathrm{x})$ Hit SE Block Change: a high score implies that as the test progresses, RT variation increases $(\mathrm{r}=0.08)$; $\mathrm{xi})$ Hit RT Interstimulus Intervals (ISI) change: a high score implies that, as the stimulus interval increased, the RTs became longer $(\mathrm{r}=0.51)$ and (xii) Hit SE ISI change: a high score implies that RT variation increased as the stimulus interval increased $(\mathrm{r}=0.05) \cdot{ }^{26}$ Some of these indices make up different variables, namely, sustained attention, inattention and vigilance.

The primary variable of interest in this study is sustained attention. This is observed through the participant's responding pattern on the CPT's hit RT block change, commissions block change and omissions block change indices. A decrease in sustained attention performance is captured by significant slowing in participant's hit RT, and significant increase in errors (omissions and commissions) as the task progresses. Participant's performance across blocks 1, 2 and 3 will be compared with their performance across blocks 4,5 and 6 of the same administration.

The indices that measure participant's inattention are poor detectability, a high number of omission and commission errors, a slow hit RT and high variability in RT (hit RT SE). The indices that measure participant's vigilance (participant's performance at different stimulus frequency or interstimulus intervals) are captured by performance on the hit RT ISI change.

\section{Secondary outcome measures Executive functioning}

A neuropsychological test battery will be used to evaluate executive attention abilities such as selective attention, attentional control, cognitive flexibility and working memory. This neuropsychological test battery comprises subtests from the WISC-IV ${ }^{97}$ and the Delis-Kaplan Executive Function System (D-KEFS) ${ }^{98}$ A parent-rated questionnaire, the BRIEF, ${ }^{99}$ will be used as a measure of day-to-day behavioural manifestations of executive functioning.

Digit Span (from the WISC-IV). ${ }^{97}$ The WISC-IV Digit Span subtest is a verbal memory task requiring participants to repeat a sequence of digits in forward and backward order. It measures a child's working memory and their ability to temporarily store information. Digit Span Forward requires the child to repeat a series of numbers given verbally by the examiner, increasing one digit per item from two digits to a maximal of nine digits. Digit Span Backward requires the child to repeat given number series in the reverse order (eg, if the examiner said ' $5-7-$ 4 ' the child should say ' $4-7-5$ '). A score of 1 will be given to each string correctly answered and a total score will be generated (Digit Span Backward range $=0-16$ ). Raw scores 
can be converted into scaled scores $(\mathrm{M}=10, \mathrm{SD}=3)$. Digit Span Backward has a good internal consistency $(\alpha=0.80)^{97}$ and a high test-retest reliability $(\mathrm{r}=0.74) .{ }^{97}$

Symbol Search (from the WISC-IV). ${ }^{97}$ The WISC-IV Symbol Search subtest will be used as a measure of selective attention. Participants will be required to visually scan a group of symbols and indicate whether a target symbol is present in the group by placing a line through the word 'yes' or 'no' for every target item on this subtest. Participant scores are calculated by taking away the total number of incorrectly identified symbols from the total number of correctly identified symbols. Symbol Search has adequate internal consistency $(\alpha=0.79)$ and a high level of testretest reliability $(\mathrm{r}=0.80){ }^{97}$

Colour-Word Interference Test (from D-KEFS). ${ }^{98}$ The inhibition condition (cond 3 ) of the Colour-Word Interference Test will be used to measure attentional control, while the inhibition/switching condition (cond 4) will be used to measure attentional control and cognitive flexibility. In the inhibition condition (cond 3), children will be required to name the ink colour that colour words are printed in. The total number of errors and the time it takes to complete the task will be used as outcome measures of attentional control, with the longer times and more errors indicating poorer attentional control. In the inhibition/switching condition (cond 4), children will be required to switch between naming the colour of the ink that the word is printed in (like in cond3) and reading the word (if it is presented in a box). The total time it takes to complete the task will be used as outcome measures of cognitive flexibility, while the total number of errors will be used as outcome measures of attentional control. The Colour-Word Interference Test has excellent test-retest reliability $(\mathrm{r}=0.79-0.90) .{ }^{100}$

Trail Making Test (D-KEFS). ${ }^{98}$ The number-letter switching condition cond 4) from the Trail Making Test will be used to measure attentional control and cognitive flexibility. In condition 4 of the Trail Making Test, children will be required to switching back and forth between connecting numbers in numerical order and letters in alphabetical order, for example, 1 to $\mathrm{A}$ to 2 to $\mathrm{B}$ to 3 to $\mathrm{C}$ and so on all the way to $\mathrm{P}$. The number of errors and the time it takes to complete the task will be recorded and the more error and the longer it takes to complete the task indicates greater difficulty with attention control and cognitive flexibility. Trail Making Test has moderate testretest reliability $(\mathrm{r}=0.20-0.82)$ has been documented. ${ }^{100}$

$B R I E F^{29}$ The BRIEF is a parent-rated questionnaire for assessing day-to-day behavioural manifestations of executive function. Parents will be required to rate 86 items (eg, 'gets stuck on one topic or activity') on a 3-poinLikertrt scale, from 1 (never) to 3 (often). The provided normative data in the manual will allow raw scores to be converted into $\mathrm{T}$ scores $(\mathrm{M}=50, \mathrm{SD}=10)$. Eight subscales are combined to form the Behavioural Regulation Index (initiate, working memory, plan/organise, organisation of materials and monitor subscales) and the Metacognition Index (inhibit, emotional control and shift subscales). Together these form the overall Global Executive Composite (GEC). T scores of 65 or more (1.5 SD above the mean) indicate elevated levels on the corresponding subscales and indices. Higher T scores indicate greater executive dysfunction. In this study, the GEC will be used as primary measures of executive function in everyday life. The BRIEF has been shown to be a valid measure of executive functioning and has good internal consistency $(\alpha=0.80-0.98)$ and high test-retest reliability on the GEC $(\mathrm{r}=0.86) .{ }^{101}$

\section{Mindfulness}

$\mathrm{CAMM}^{73}$ assesses present-moment awareness and non-judgmental, non-avoidant responses to thoughts and feelings. In this 10-item questionnaire, children are asked to indicate how each item reflects their experience using a 5-point Likert scale ranging from 0 (never true) to 4 (always true). A total acceptance-mindfulness score will be generated by reverse scoring negatively worded items and summing the item total, yielding a possible range in scores from 0 to 100 . The higher scores indicate higher levels of acceptance and mindfulness. This test has been shown to have good internal consistency $(\alpha=0.81) .^{73}$

\section{Behavioural}

$\mathrm{SDQ}^{71}{ }^{102}$ is a 25-item parent-report questionnaire designed to assess their child's behaviour and adjustment. The items are divided into five scales to assess the frequency within the last 6 months of emotional symptoms, conduct problems, inattention/hyperactivity, peer problems and prosocial behaviour. These items are rated on 3-point Likert scale, from 0 (not true) to 2 (certainly true), allowing for a total score for each scale $(0-10)$ to be calculated, along with an overall difficulties score that takes into account overlapping elements of each score $(0-40)$. Higher scores indicate more distress on all scales except prosocial behaviour. In this study, the SDQ total score will be used as primary measures of children's behaviour. SDQ total score has been shown to have a moderate to high level of internal consistency $(\alpha=0.73-$ $0.82)^{103104}$ and a moderate-to-high test-retest reliability $(\mathrm{r}=0.77-0.85){ }^{102} 104$

\section{Physical outcome measures \\ Flexibility}

Lower back and hamstring muscle flexibility will be assessed by the sit and reach test (reach distance). Participants sit on the floor with both legs extended as straight as possible in front, heels placed on the floor and feet flat against a wall so that the ankle is dorsiflexed to approximately $90^{\circ}$. Participants are instructed to stretch their arms straight in front with hands overlapping (middle fingers overlapping too) and slowly bend forward at the hip joint reaching as far as possible towards their toes. The maximum reach must be held for $2 \mathrm{~s}$, and the distance between the tips of the middle fingers to the wall at the top of the toes is recorded. 
Lower limb functional strength

The following functional exercises will be used to assess strength:

Lateral step-up. Lateral step-up records the number of steps up on to a step from the floor that the child can perform during $30 \mathrm{~s} .^{72}$ The test will be performed on a $10 \mathrm{~cm}$ high step for all children, with a complete up and down step being one full cycle. The child stands parallel to the step with the leg being tested on the step, and the non-testing leg on the floor. They are asked to extend their test leg (on the step) straight and lift their non-test leg off the ground and onto the step and then lower the non-test leg back down to the floor until the foot touches the ground. ${ }^{105}$ This is then repeated for the other leg. Reliability for this test is strong (intraclass correlation $(\mathrm{ICC})=0.94$ ) and mean repetitions for the lateral step up were $13.2(\mathrm{SD}=10.5 ; \mathrm{SEM}=2.4$ reps; coefficient of variation $(\mathrm{CV})=17.8 \%)$ for the left side, and $12.6(\mathrm{SD}=10.4 ; \mathrm{SEM}=2.6 \mathrm{reps} ; \mathrm{CV}=22.7 \%)$ for the right side. ${ }^{72}$

Sit-to-stand. The sit-to-stand functional strength test records the number of sit-stand-sit repetitions that the child can perform within $30 \mathrm{~s} .{ }^{72}$ The test will be performed on a chair where the child's knees and hips are at $90^{\circ}$ flexion, upper legs parallel to the floor and feet flat on the floor. The child will be asked to stand up straight so that their hips and knees are extended and then sit back down. Reliability for this test was strong (ICC=0.91) and the mean number of repetitions was $14.4(\mathrm{SD}=5.0$; $\mathrm{SEM}=2.6$ reps; $\mathrm{CV}=22.7 \%) .^{72}$

Half-kneel to stand. Half-kneel to stand records the number of repetitions from half kneel to stand the child can perform within $30 \mathrm{~s}^{72}$ The child is positioned on a mat, with one leg kneeling on the floor while the other leg is in front with the knee at $90^{\circ}$ flexion and foot flat on the floor. From this half-kneeling position, the child is asked to assume a standing position. Repetitions are counted each time the participant achieves a standing position where both legs and hips are extended as much as possible. This is then repeated with the other leg in front. Reliability for this test was strong ( $\mathrm{ICC}=0.93-0.96$ ) and mean repetitions were 7.5 reps $(\mathrm{SD}=5.5 ; \mathrm{SEM}=1.1$ reps; $\mathrm{CV}=28.6 \%)$ for the left side and $6.0 \quad(\mathrm{SD}=5.3$; $\mathrm{SEM}=1.4$ reps; $\mathrm{CV}=39.9 \%$ ) for the right side. ${ }^{72}$

Submaximal motor capacity test. The $6 \mathrm{~min}$ walk test $(6 \mathrm{MWT})^{106}$ is submaximal clinical exercise test which measures the distance the child can walk in 6 min. ${ }^{106}$ The child will be asked to walk as many laps as they can without running for $6 \mathrm{~min}$ along a straight, flat a corridor, between two markers set $10 \mathrm{~m}$ apart. The $6 \mathrm{MWT}$ will be conducted with standardised verbal encouragement and the child will be advised on the time remaining every minute and the number of laps they have completed as it happens. The $6 \mathrm{MWT}$ has been demonstrated to be reliable in independently ambulant adolescents with CP and the test-retest is excellent $(\mathrm{ICC}=0.98) .{ }^{106}$
Mobility

The MobQues28 questionnaire measures mobility limitations a child with CP experiences in everyday life as rate by their parents. ${ }^{107} 108$ The MobQues28 is score on a5-point Likert scale from 0 to 4 , with 0 being impossible without help; 1 being very difficult; 2 being somewhat difficult; 3 being slightly difficult and 4 being not difficult at all. Item scores (range 0-4) are added together and then divided by the maximum possible score and multiplied by 100 to obtain total scores on a scale of 0-100 (lower scores indicate severe limitations in mobility $)$ : MobQues28 $=(\Sigma$ item/112) 100 . Construct validity was demonstrated as MobQues28 scores decreased with increasing GMFCS level $(\mathrm{p}<0.001)$ and in a subgroup of 162 children, MobQues28 score was positively correlated to GMFM-66 $(\mathrm{r}=0.67, \mathrm{p}<0.001) .{ }^{108}$ The MobQues28 has been demonstrated to be reliable with high inter-rater reliability (ICC=0.87), SEM was 8.9. The intra-rater reliability was higher $(\mathrm{ICC}=0.96-0.97 ; \mathrm{SEM}=4.4-4.9){ }^{107}$

\section{Quality of life}

CP QOL-Child. The CP QOL-Child is a condition-specific quality of life measure for children with $\mathrm{CP}^{74}$ Two versions of the CP QOL-Child will be used in this study; the CP QOL-Child parent report version will be used for all participants aged under 12 years, and the CP QOL-Child self-report version will be used for children aged 9-12 years. The parent-report measure of child quality of life is a 66-item questionnaire and the child self-report version is a 53-item questionnaire. Results of factor analysis demonstrated that the CP QOL measures seven broad domains of quality of life: social well-being and acceptance, functioning, participation, physical health, emotional well-being, access to services, pain, impact of disability and family health. In this study, the GEC will be used as primary measures of executive function in everyday life. The CP QOL-Child has a high internal consistency $(\alpha=0.74-0.92$ for parent report and $\alpha=0.80-0.90$ for child self-report), good test-retest reliability $(\mathrm{ICC}=0.76-0.89)$ and it is moderately correlated with generic QOL and health measures $(\mathrm{r}=0.30-0.51) .^{109110}$

CP QOL-Teen. The CP QOL-Teen is a condition-specific quality of life measure for adolescents with $\mathrm{CP} .^{75}$ Two versions of this CP-QOL will also be used: the CP QOL-Teen parent report version will be completed by parents of participants aged 13-18 years and the CP QOL-Teen self-report version will be completed by participates aged $13-18$ years. ${ }^{75}$ This measure has strong internal consistency $(\alpha=0.81-0.95$ for the primary parent report, and $\alpha=0.84-0.96$ for the adolescent self-report), good test-retest reliability for primary parents ( $\mathrm{ICC}=0.72-0.92)$ and adolescents $(\mathrm{ICC}=0.84-0.87)$. All domains of the $\mathrm{CP}$ QOL-Teen parent report $(\mathrm{r}=0.40-46)$ and adolescent report $(r=0.58-0.68)$ were correlated with a generic $Q O L$ instruments. $^{111}$ 


\section{Pain}

The Baker-Wong Faces Pain Rating Scale will be used to assess the child's pain level. ${ }^{112}$ Children will be asked to match which of the six hand-drawn faces show their pain level, with faces ranging from smiling ('no hurt') to crying ('hurts worst'), with the scale scored from 0 to 5 . It has been documented that Baker-Wong Faces Pain Rating Scale has an excellent test-retest reliability over $15 \mathrm{~min}$, 8 hours and immediately post-procedure in children aged 3-18 years $(\mathrm{r}=0.90-0.84) .{ }^{113} 114$ There are also strong positive correlations between the Baker-Wong Faces Pain Scale and other well-established self-report measures $(\mathrm{r}=0.74-0.78)$ (eg, pieces of hurt tool, Faces Pain Scale and a Visual Analogue Scale). ${ }^{113} 115-117$

\section{Parent outcome measures}

The following tests will be used to assess the psychological well-being of parents. These assessments will be completed at baseline, pre-intervention, post-intervention and at a 6-month follow-up.

\section{Mindfulness Attention Awareness Scale ${ }^{76}$}

MAAS will be used to measure each parents' tendency to be mindful of moment-to-moment experiences. ${ }^{76}$ The MAAS is a 15-item instrument focusing on the presence or absence of attention and awareness of their present immediate environment. Participants are asked to indicate how frequently they have the experience described in each of the 15 statements using a 6-point Likert scale from 1 (almost always) to 6 (almost never). For example, one statement is: "It seems I am 'running on automatic', without much awareness of what I'm doing", while another is: "I snack without being aware that I am eating". The total score is used as outcome measure, with higher scores reflecting more mindfulness. This scale has been shown to relate to various aspects of well-being and to how effectively people deal with stressful life events. ${ }^{76}$ The MAAS also has good internal consistency levels generally ranging from 0.80 to $0.90 .^{76}$

\section{Acceptance and Action Questionnaire ${ }^{78}$}

The AAQ assesses the construct referred to as acceptance, experiential avoidance or psychological inflexibility. ${ }^{118}$ This 7-item questionnaire has been documented to have a satisfactory structure, reliability and validity, with a mean $\alpha$ coefficient of $0.84(0.78-0.88)$ and the 3-month and 12 -month test-retest reliability is 0.81 and 0.79 , respectively. ${ }^{118}$

\section{Depression Anxiety Stress Scale ${ }^{77}$}

DASS is a 42-item questionnaire that assesses the presence of psychological symptoms in adults. ${ }^{77}$ In this study, a total score will be computed, falling in the range 0-126, with higher scores indicating suboptimal states. The measure has shown high internal consistency for depression $(\alpha=0.91)$, anxiety $(\alpha=0.84)$ and stress $(\alpha=0.90)$ scales. DASS also has good discriminate and concurrent validity. ${ }^{77} 119$
Personal Well-being Index-Adult ${ }^{79}$

The PWI-A is an 8-item questionnaire that assesses the ones' level of satisfaction with life as a whole. ${ }^{79}$ Each item corresponds to a quality of life domain, such as standard of living, health, achieving in life, relationships, safety, community-connectedness, future security and spirituality/religion.

\section{Child-Parent Relationship Scale ${ }^{80}$}

The CPRS is a 15-item questionnaire that uses a 5-point rated scale and it is completed by the parent to assess the quality of the parent-child relationship. ${ }^{80} 120$ The CPRS is an adaptation of the Student-Teacher Relationship Scale, which has been used extensively in studies of relationship quality in the USA (National Institute of Child Health and Development Early Child Care Research Network. ${ }^{121}$ In this study, the overall conflict score and closeness score will be used as measures of parent-child relationship quality.

\section{MiYoga session measures}

The following tests have been developed in order to measure the mechanism of change due to the MiYoga intervention. Both child and parent participants will complete these measures either before and after or just after each MiYoga session.

\section{Mindfulness}

MiYoga Session Mindfulness Measure is a 5-item scale adapted from the CAMM. It measures children and parent's mindfulness in the MiYoga session they have just completed. Each child and parent will complete this mindfulness scale at the end of each MiYoga session (see online supplementary appendix E).

\section{Mood}

The Mood Scale is a short scale used to measure participants' mood or how they are feeling before and after each MiYoga session. It is a 5-iteLikertrt scale with 5 being very good and 1 being very bad, where 5 is matched with a happy face, 3 is matched with a neutral face and 1 is matched with a sad face (see online supplementary appendix F).

\section{Session feedback}

At the end of each MiYoga session, children and parents will each be asked to complete a Session Feedback Scale. This is a short four-item questionnaire asking the participants about the session in regard to comfort, enjoyment, body awareness and overall satisfaction. Each of these questions is also on a $0-10$ scale, with 10 being high (or very good) and 0 being low (or very bad; see online supplementary appendix G).

\section{Qualitative interviews}

A registered psychologist (or a probationary psychologist under supervision) will conduct a short interview with individual participants at the end of the MiYoga programme. The interviewer will be unrelated to the MiYoga study to 
avoid bias, and to encourage participants to express their view and opinions about the programme freely. The aim of these interviews is to gather qualitative information about the participants' views of the programme, such as what they liked or disliked, and which parts worked (or not) for them. This qualitative information will help with programme refinement and translation. For example, it will provide information on how mindfulness and yoga can be integrated into participants' everyday life (see online supplementary appendix $\mathrm{H}$ for qualitative interview script and questions).

\section{STATISTICAL ANALYSIS}

\section{Sample size}

The reaction time of children with CP was 2 SD below healthy controls on the (CPT). ${ }^{9}$ The present study is interested in detecting a difference of 1 SD between the immediate treatment and control group at time 2 (post-intervention) to determine the clinical significance of the MiYoga programme compared with waitlist control. ${ }^{122}$ Based on Lehr's equation, with alpha set at 0.05 , and power set at 0.80 , to detect a difference of 1 SD between groups, a sample size of 16 participants per group would be needed. ${ }^{123}$ If a retention rate of $90 \%$ is assumed, a total of 36 children will need to be recruited.

\section{Randomised controlled trial data analysis}

Analysis will follow standard principles for randomised controlled trials, using two-group comparisons on all participants on an intention-to-treat basis. All the participants will be analysed within the group they were randomised for all the outcome measures. Any missing data in the event of withdrawal or loss to follow-up, a participant's assessment from the last available time-point will be carried forward. External and internal validity of results will be checked using baseline and general descriptive information available for all eligible families. This will be done by comparing the characteristics of families who completed the study with those who enrolled in the study but did not complete, and those who did not enrol. The primary comparison immediately post-intervention (T2) will be scores from the CPT.

Analysis will follow standard methods for randomised controlled trials using comparisons between the two groups (MiYoga and waitlist control). The experimental unit will be the child and his/her participating parent. Attrition analysis will be conducted. Data will be analysed using Statistical Package for the Social Sciences version 24. The hypotheses relating to intervention efficacy will be tested using general linear models, specifically via analysis of covariance. In addition, predictors of treatment outcome will be explored using regression analyses. Non-parametric methods will be used for simple comparisons in cases where continuous data exhibit substantial skewness not overcome by transformation.

\section{Qualitative data analysis}

A descriptive thematic analysis will be undertaken to analyse the qualitative data collected in this study (as per Braun and Clarke ${ }^{124}$ ). The interviews will be audio-recorded and transcribed verbatim. Transcripts will be read and coded by two study investigators. Coding will involve identifying thematic patterns, such as ideas, concepts, terminology or phrases. Once these themes are identified, the investigators will discuss the appropriateness of the themes and develop a coding framework based on their themes until consensus is achieved. The researchers will then reread the transcripts and organise the themes into categories and subcategories based on the coding framework they have developed.

\section{ETHICS AND DISSEMINATION}

Full ethical approval for this study has been obtained by the Behavioral and Social Sciences Ethical Review Committee of The University of Queensland (2012000993) and Children's Health Queensland Hospital and Health Service Research Ethics Committee (HREC/12/QRCH/120). Protocol modifications and amendments will be submitted to the ethical committees for approval. This trial has been registered with the Australian New Zealand Clinical Trials Registry (ACTRN12613000729729). All families will be given written informed consent to participate and their parents or guardians prior to entering into the trial. If found to be effective, MiYoga has the potential to be disseminated as a lifestyle intervention for families. The study results will be disseminated through publication in scientific journals, presentation at relevant conferences and directly to the families who participated in the study. The dissemination would assist children with $\mathrm{CP}$ and complement their ongoing therapy by improving the ability of the child to pay attention at school and in therapy, and alleviating environmental stressors for both the child and their parents.

\section{DISCUSSION}

This paper has presented the background and design for a randomised waitlist controlled trial investigating the efficacy of an 8-week mindfulness and yoga programme for children and adolescents with a CP and one of their parents. To our knowledge, this study is the first to investigate the effects of a family-centred mindfulness-based yoga programme for children and adolescents with CP. Furthermore, we will be evaluating the children's cognitive, physical, behaviour and emotional outcomes, as well as parents' psychological well-being. The qualitative information, such as what the participants liked or disliked about the programme, will help provide information on how mindfulness and yoga can be integrated effectively into participants' everyday lives. It is anticipated that the results of this study will be disseminated through peer-reviewed journals and national and international academic conferences. 
Current models of rehabilitation for children with CP solely focus on physical rehabilitation. MiYoga provides a more holistic approach by combining mindfulness and yoga techniques that can be integrated easily into the child and their families' everyday life. If MiYoga is found to be effective, multidisciplinary teams will have an evidence-based mode of therapy to offer, that is, an engaging lifestyle intervention, incorporates cognitive rehabilitation and provides valuable tools and skills to families.

Correction notice This article has been corrected since it first published. Several typos throughout the text have been corrected. These changes did not affect the data in the article.

Contributors $\mathrm{CM}$ is the chief investigator and together with $\mathrm{KW}$ and $\mathrm{RB}$ designed and established this research study. CM was responsible for ethics applications and reporting. $\mathrm{CM}$ was responsible for recruitment. $\mathrm{CM}$ was responsible for data collection and implementation of the training programme. CM will take lead roles on preparation for publications on the clinical outcomes of the study. KW, RB and $\mathrm{RC}$ will contribute to the preparation of publications within their respective fields of expertise. CM will take on a lead role of the statistical analysis for the study. $\mathrm{CM}$ drafted the final version of this manuscript. All authors critically reviewed and approved the final version. All data from this study will be submitted to peer-review journals. CM will use the data from this study to contribute to her PhD thesis.

Funding CM is a PhD scholar funded by The University of Queensland Research Scholarship. KW is a Postdoctoral Fellow funded by the National Health and Medical Research Council (NHMRC; grant number 631712). RB is a Research Fellow also funded by the NHMRC (grant number 1105038). This work was also supported by Merchant Charitable Foundation through Children's Health Queensland.

Competing interests MiYoga was developed by first author.

\section{Patient consent None.}

Ethics approval Full ethical approval for this study has been obtained by the Behavioral and Social Sciences Ethical Review Committee of The University of Queensland (2012000993) and Children's Health Queensland Hospital and Health Service Research Ethics Committee (HREC/12/QRCH/120). All families will be given written informed consent to participate and their parents or guardians prior to entering into the trial.

\section{Provenance and peer review Not commissioned; externally peer reviewed.}

Data sharing statement In accordance with the protocol, the data of the study will be published.

Open Access This is an Open Access article distributed in accordance with the Creative Commons Attribution Non Commercial (CC BY-NC 4.0) license, which permits others to distribute, remix, adapt, build upon this work non-commercially, and license their derivative works on different terms, provided the original work is properly cited and the use is non-commercial. See: http://creativecommons.org/ licenses/by-nc/4.0/

(c) Article author(s) (or their employer(s) unless otherwise stated in the text of the article) 2017. All rights reserved. No commercial use is permitted unless otherwise expressly granted.

\section{REFERENCES}

1. Sellier E, Platt MJ, Andersen GL, et al. Decreasing prevalence in cerebral palsy: a multi-site European population-based study, 1980 to 2003. Dev Med Child Neurol 2016;58:85-92.

2. Rosenbaum P, Paneth N, Leviton A, et al. A report: the definition and classification of cerebral palsy April 2006. Dev Med Child Neurol Suppl 2007;109:8-14.

3. Straub K, Obrzut JE. Effects of cerebral palsy on neuropsychological function. J Dev Phys Disabil 2009;21:153-67.

4. Novak I, Hines M, Goldsmith S, et al. Clinical prognostic messages from a systematic review on cerebral palsy. Pediatrics 2012;130:E12 85-E312.

5. Nielsen HH. Psychological appraisal of children with cerebral plasy: a survey of 128 re-assessed cases. Dev Med Child Neurol 1971;13:707-20.
6. Novak I, Mclntyre S, Morgan C, et al. A systematic review of interventions for children with cerebral palsy: state of the evidence. Dev Med Child Neurol 2013;55:885-910.

7. Australian cerebral palsy register report 2009, birth years 19932003. Canberra: The Australian Cerebral Palsy Register Group, 2009.

8. Bottcher L, Flachs EM, Uldall P. Attentional and executive impairments in children with spastic cerebral palsy. Dev Med Child Neurol 2010;52:e42-e47.

9. Lemay M, Lê T-T, Lamarre C. Deficits in two versions of a sustained attention test in adolescents with cerebral palsy. Dev Neurorehabil 2012;15:253-8.

10. Whittingham K, Bodimeade HL, Lloyd O, et al. Everyday psychological functioning in children with unilateral cerebral palsy: does executive functioning play a role? Dev Med Child Neurol 2014;56:572-9.

11. Diamantopoulou S, Rydell AM, Thorell LB, et al. Impact of executive functioning and symptoms of attention deficit hyperactivity disorder on children's peer relations and school performance. Dev Neuropsychol 2007;32:521-42.

12. Posner MI, Petersen SE, Posner PSE. The attention system of the human brain. Annu Rev Neurosci 1990;13:25-42.

13. Theeuwes J. Exogenous and endogenous control of attention: the effect of visual onsets and offsets. Percept Psychophys 1991;49:83-90.

14. Posner MI, Rothbart MK. Attention, self-regulation and consciousness. Philos Trans R Soc Lond B Biol Sci 1998;353:1915-27.

15. Posner MI. Orienting of attention. Q J Exp Psychol 1980;32:3-25.

16. Astle DE, Scerif $G$. Using developmental cognitive neuroscience to study behavioral and attentional control. Dev Psychobiol 2009;51:107-18.

17. Rueda MR, Rothbart MK, McCandliss BD, et al. Training, maturation, and genetic influences on the development of executive attention. Proc Natl Acad Sci U S A 2005;102:14931-6.

18. Robertson IH, Garavan H. Vigilant attention In: M.S. G, ed. The cognitive neurosicences. 2nd ed. Cambridge: MIT Press, 2004:631-40.

19. Dally K. The influence of phonological processing and inattentive behavior on reading acquisition. J Educ Psychol 2006;98:420-37.

20. Dobbs J, Doctoroff GL, Fisher PH, et al. The association between preschool children's socio-emotional functioning and their mathematical skills. J Appl Dev Psychol 2006;27:97-108.

21. Fuchs LS, Fuchs D, Compton DL, et al. The cognitive correlates of third-grade skill in arithmetic, algorithmic computation, and arithmetic word problems. J Educ Psychol 2006;98:29-43.

22. Warner-Rogers J, Taylor A, Taylor E, et al. et allnattentive behavior in childhood: epidemiology and implications for development. $J$ Learn Disabil 2000;33:520-36.

23. Meyer ML, Lieberman MD. Social working memory: neurocognitive networks and directions for future research. Front Psychol 2012;3:571.

24. Robertson IH, Ridgeway V, Greenfield E, et al. Motor recovery after stroke depends on intact sustained attention: a 2-year follow-up study. Neuropsychology 1997;11:290-5.

25. Odding E, Roebroeck ME, Stam HJ. The epidemiology of cerebral palsy: incidence, impairments and risk factors. Disabil Rehabil 2006;28:183-91.

26. Conners' Continuous Performance Test (CPT II): version 5 for Windows: technical guide and software Manual. MHS, 2004.

27. Wechsler D. WISC-III: wechsler intelligence scale for children: manual. Psychological Corporation, 1991.

28. Manly TM, Robertson IH, Anderson V, et al. TEA-Ch: test of Everyday attention for Children. London: Pearson Assessment, 1999.

29. GioiaB. behavior rating inventory of executive function: professional manual. Psychological Assessment Resources, 2000.

30. Semrud-Clikeman M, Nielsen $\mathrm{KH}$, Clinton $\mathrm{A}$, et al. An intervention approach for children with teacher- and parent-identified attentional difficulties. J Learn Disabil 1999;32:581-90.

31. Williams DJ. A process-specific training program in the treatment of attention deficits in children. Seattle: University of Washington, 1989.

32. Thomson JB. Rehabilitation of individuals with traumatic brain injury through utilizationof an attention training program. 23rd Annual International NeuropsychologicalSociety Conference. Seattle, WA, 1995.

33. Butler RW, Namerow NS. Review article: cognitive retraining in brain-injury rehabilitation: a critical review. Neurorehabil Neural Repair 1988;2:97-101. 
34. Bishop SR, Lau M, Shapiro S, et al. Mindfulness: a proposed operational definition. Clinical Psychology: Science and Practice 2004;11:230-41.

35. Lutz A, Slagter HA, Dunne JD, et al. Attention regulation and monitoring in meditation. Trends Cogn Sci 2008;12:163-9.

36. Hasenkamp W, Wilson-Mendenhall CD, Duncan E, et al. Mind wandering and attention during focused meditation: a fine-grained temporal analysis of fluctuating cognitive states. Neuroimage 2012;59:750-60.

37. Jha AP, Krompinger J, Baime MJ. Mindfulness training modifies subsystems of attention.cognitive, affective, \&. Behav Neurosci 2007;7:109-19.

38. Tang YY, Ma Y, Wang J, et al. Short-term meditation training improves attention and self-regulation. Proc Natl Acad Sci U S A 2007;104:17152-6.

39. Fan J, McCandliss BD, Sommer T, et al. Testing the efficiency and independence of attentional networks. J Cogn Neurosci 2002;14:340-7.

40. Kabat-Zinn J. Mindfulness-Based Interventions in Context: Past, Present, and Future. Clinical Psychology: Science and Practice 2003;10:144-56.

41. Fronske HA. Teaching cues for sport skills for secondary school students. 3 ed. San Francisco, CA: Pearson Education, Inc, 2005.

42. Desikachar TKV. The Heart of Yoga: developing a personal practice Rochester. Vermont: Inner Traditions International, 1995.

43. Desikachar TKV. Reflections on Yoga Sutras of Patanjali. India: Krishnamacharya Yoga Mandiram, 2003.

44. Manjunath NK, Telles S. Spatial and verbal memory test scores following yoga and fine arts camps for school children. Indian $J$ Physiol Pharmacol 2004;48:353-6.

45. Uma K, Nagendra HR, Nagarathna R, et al. The integrated approach of yoga: a therapeutic tool for mentally retarded children: a one-year controlled study. J Ment Defic Res 1989;33 (Pt 5:415-21.

46. Manjunath NK, Telles S. Improved performance in the tower of London test following yoga. Indian J Physiol Pharmacol 2001;45:351-4

47. Jensen PS, Kenny DT. The effects of yoga on the attention and behavior of boys with Attention-Deficit/ Hyperactivity Disorder (ADHD). J Atten Disord 2004;7:205-16.

48. Dash M, Telles S. Improvement in hand grip strength in normal volunteers and rheumatoid arthritis patients following yoga training Indian J Physiol Pharmacol 2001;45:355-60.

49. Dhume RR, Dhume RA. A comparative study of the driving effects of dextroamphetamine and yogic meditation on muscle control for the performance of balance on balance board. Indian J Physiol Pharmacol 1991;35:191-4.

50. Mandanmohan Jatiya L Udupa K et al et a/Effect of yoga training on handgrip, respiratory pressures and pulmonary function Indian $J$ Physiol Pharmacol 20034738792

51. Telles S, Hanumanthaiah B, Nagarathna R, et al. Improvement in static motor performance following yogic training of school children. Percept Mot Skills 1993;76:1264-6.

52. Kabat-Zinn J. University of Massachusetts Medical Center/ Worcester. Stress Reduction C. full catastrophe living: using the wisdom of your body and mind to face stress, pain, and illness. New York: Dell Publishing, 1991.

53. Carmody J, Baer RA, L B Lykins E, et al. An empirical study of the mechanisms of mindfulness in a mindfulness-based stress reduction program. J Clin Psychol 2009;65:613-26.

54. Grossman P, Niemann L, Schmidt S, et al. Mindfulness-based stress reduction and health benefits. A meta-analysis. J Psychosom Res 2004;57:35-43.

55. Mak C, Whittingham $\mathrm{K}$, Cunnington R, et al. Efficacy of mindfulness-based interventions for attention and executive function in children and adolescents - a systematic review. Mindfulness under review.

56. Britton WB, Lepp NE, Niles HF, et al. A randomized controlled pilot trial of classroom-based mindfulness meditation compared to an active control condition in sixth-grade children. J Sch Psychol 2014;52:263-78.

57. Felver JC, Tipsord JM, Morris MJ, et al. The effects of mindfulnessbased intervention on children's attention regulation. J Atten Disord 2014.

58. Flook L, Smalley SL, Kitil MJ, et al. Effects of Mindful Awareness Practices on Executive Functions in Elementary School Children. J Appl Psychol 2010;26:70-95.

59. Haffner J, Roos J, Goldstein N, et al. [The effectiveness of bodyoriented methods of therapy in the treatment of attention-deficit hyperactivity disorder (ADHD): results of a controlled pilot study]. $Z$ Kinder Jugendpsychiatr Psychother 2006;34:37-47.
60. Kratter J. The use of meditation in the treatment of attention deficit disorder with hyperactivity. ProQuest Dissertations Publishing, 1983.

61. Leonard NR, Jha AP, Casarjian B, et al. Mindfulness training improves attentional task performance in incarcerated youth: a group randomized controlled intervention trial. Front Psychol 2013;4:792.

62. Moretti-Altuna $\mathrm{G}$. The effects of meditation versus medication in the treatment of attention deficity disorder with hyperactivity (relaxation training, impulsivity, ritalin. ProQuest Dissertations Publishing, 1987.

63. Purohit SP, Pradhan B. Effect of yoga program on executive functions of adolescents dwelling in an orphan home: a randomized controlled study. J Tradit Complement Med 2017;7:99-105.

64. Schonert-Reichl KA, Oberle E, Lawlor MS, et al. Enhancing cognitive and social-emotional development through a simpleto-administer mindfulness-based school program for elementary school children: a randomized controlled trial. Dev Psychol 2015:51:52-66.

65. Semple RJ, Lee J, Rosa D, et al. A randomized trial of mindfulnessbased cognitive therapy for children: promoting mindful attention to enhance social-emotional resiliency in children. IJCYFS 2010;19:218-29.

66. Sidhu P. The efficacy of mindfulness meditation in increasing the attention span in children with $A D H D$. ProQuest Information \& Learning, 2015.

67. Telles S, Singh N, Bhardwaj AK, et al. Effect of yoga or physical exercise on physical, cognitive and emotional measures in children: a randomized controlled trial. Child Adolesc Psychiatry Ment Health 2013;7:37.

68. Verma IC, Jayashankarappa BS, Palani M. Effect of transcendental meditation on the performance of some cognitive psychological tests. Indian J Med Res 1982;76:136-43.

69. Rani NJ, Rao PVK. Body awareness and yoga training. Percept Mot Skills 1994;79:1103-6.

70. Tsao JC, Meldrum M, Kim SC, et al. Treatment preferences for CAM in children with chronic pain. Evid Based Complement Alternat Med 2007;4:367-74.

71. Goodman R. The strengths and difficulties Questionnaire: a research note. J Child Psychol Psychiatry 1997;38:581-6.

72. Verschuren $\mathrm{O}$, Ketelaar M, Takken T, et al. Reliability of hand-held dynamometry and functional strength tests for the lower extremity in children with cerebral palsy. Disabil Rehabil 2008;30:1358-66.

73. Greco LA, Baer RA, Smith GT. Assessing mindfulness in children and adolescents: development and validation of the Child and Adolescent Mindfulness Measure (CAMM). Psychol Assess 2011;23:606-14.

74. Waters E, Davis EB R, Reddihough D, et al. Cerebral Palsy Quality of Life Questionnaire for Children (CP QOL-Child) Manual. Melbourne: University of Melbourne, 2006.

75. Davis E, Davern M, Waters E, et al. Cerebral Palsy Quality of Life Questionnaire for adolescents (CP QOL-Teen) Manual. Melbourne: University of Melbourne, 2013.

76. Brown KW, Ryan RM. The benefits of being present: mindfulness and its role in psychological well-being. 2003;84:822-48.

77. Lovibond SH, Lovibond PF. Manual for the depression anxiety stress scales. 2nd ed. Sydney: edPsychology Foundation of Australia, 1996.

78. Hayes SC, Strosahl K, Wilson KG, et al. Measuring experiential avoidance: a preliminary test of a working model. Psychological Record 2004;54:553.

79. International Wellbeing Group. Personal Wellbeing Index. Deakin University: Melbourne: Australian Centre on Quality of Life, 2006.

80. Pianta RC. Child-Parent relationship Scale: university of Virginia. 1992.

81. Bersma D, Visscher M. Yoga Games for Children: fun and Fitness with Postures, movements and breath. United States: Hunter House Publishers, 2003.

82. Hanh TN. Planting seeds: practicing Mindfulness with Children. United States: Parallax Press, 2011

83. Nagaraja D. Buddha at Bedtime: tales of love and wisdom for you to read with your child to enchant Enlighten and Inspire. United Kingdom: Duncan Baird, 2008.

84. Radojevic N. A Mindfulness Activity Workbook for Children With Cancer. ProQuest Dissertations Publishing, 2014.

85. Reid EG. A mindfulness workbook for young children: a classroom feasibility trial. ProQuest Dissertations Publishing, 2009.

86. Reid EG, Seymour NB. Mack's Top Secret Detective Manual. United States: Lulu.com, 2011.

87. Saraswati N. Yoga Education For Children, Volume 2. India: Bihar School of Yoga, 2010.

88. Saraswati S. Yoga Education For Children, Volume 1. India: Bihar School of Yoga, 1999. 
89. Solis S. Storytime Yoga: teaching yoga to Children through Story. United States: The Mythic Yoga Studio, 2006.

90. Sumar S. Yoga for the special child: a therapeutic approach for infants and children with down syndrome, Cerebral Palsy, Autism Spectrum Disorders and Learning Disabilities. United States: Special Yoga Publications, 2007.

91. Williams N. Yoga therapy for every special child. United Kingdom: Singing Dragon, Jessica Kingsley Publishers, 2010.

92. Palisano R, Rosenbaum P, Walter S, et al. Development and reliability of a system to classify gross motor function in children with cerebral palsy. Dev Med Child Neurol 1997;39:214-23.

93. Palisano RJ, Hanna SE, Rosenbaum PL, et al. Validation of a model of gross motor function for children with cerebral palsy. Phys Ther 2000;80:974-85.

94. Morris C, Galuppi BE, Rosenbaum PL. Reliability of family report for the gross motor function classification system. Dev Med Child Neurol 2004;46:455-60.

95. Crawford JR, Anderson V, Rankin PM, et al. An index-based shortform of the WISC-IV with accompanying analysis of the reliability and abnormality of differences. Br J Clin Psychol 2010;49:235-58.

96. Wechsler D. Wechsler Intelligence Scale for Children - fourth edition: technical and interpretative manual. San Antonio: The Psychological Corporation, 2003.

97. Wechsler D. Wechsler Intelligence Scale for Children - fourth edition: administration and scoring manual. New York: Psychological Corporation, 2004

98. Delis DC, Kaplan E, Kramer JH. Delis-Kaplan Executive function system (D-KEFS) examiner's manual. San Antonio: The Psychological Corporation, 2001.

99. Gioia G, Isquith P, Guy S, et al. Behavior Rating Inventory of Executive function. Odessa: Psychological Assessment Resources, 2000.

100. Delis DC, Kaplan E, Kramer JH. Delis-Kaplan Executive function system (D-KEFS) technical manual. San Antonio, Tx: The Psychological Corporation, 2001.

101. Baron IS. Behavior rating inventory of executive function. Child Neuropsychol 2000;6:235-8.

102. Goodman R. The extended version of the strengths and difficulties questionnaire as a guide to child psychiatric caseness and consequent burden. J Child Psychol Psychiatry 1999;40:791-9.

103. Goodman R. Psychometric properties of the strengths and difficulties questionnaire. J Am Acad Child Adolesc Psychiatry 2001;40:1337-45.

104. Hawes DJ, Dadds MR. Australian data and psychometric properties of the strengths and difficulties questionnaire. Aust N Z J Psychiatry 2004;38:644-51.

105. Worrell TW, Crisp E, Larosa C. Electromyographic reliability and analysis of selected lower extremity muscles during lateral step-up conditions. J Athl Train 1998;33:156-62.

106. Maher CA, Williams MT, Olds TS. The six-minute walk test for children with cerebral palsy. Int J Rehabil Res 2008;31:185-8.
107. Van Ravesteyn NT, Dallmeijer AJ, Scholtes VA, et al. Measuring mobility limitations in children with cerebral palsy: interrater and intrarater reliability of a mobility questionnaire (MobQues). Dev Med Child Neurol 2010;52:194-9.

108. van Ravesteyn NT, Scholtes VA, Becher JG, et al. Measuring mobility limitations in children with cerebral palsy: content and construct validity of a mobility questionnaire (MobQues). Dev Med Child Neurol 2010;52:e229-e235.

109. Davis $E$, Waters $E$, Mackinnon A, et al. Paediatric quality of life instruments: a review of the impact of the conceptual framework on outcomes. Dev Med Child Neurol 2006;48:311-8.

110. Waters E, Davis E, Mackinnon A, et al. Psychometric properties of the quality of life questionnaire for children with CP. Dev Med Child Neurol 2007;49:49-55.

111. Davis E, Mackinnon A, Davern M, et al. Description and psychometric properties of the CP QOL-Teen: a quality of life questionnaire for adolescents with cerebral palsy. Res Dev Disabil 2013;34:344-52.

112. Wong DL, Baker CM. Pain in children: comparison of assessment scales. Pediatr Nurs 1988;14:9-17.

113. Gharaibeh $\mathrm{M}, \mathrm{Abu}-\mathrm{Saad} \mathrm{H}$. Cultural validation of pediatric pain assessment tools: Jordanian perspective. J Transcult Nurs 2002:13:12-18.

114. Keck JF, Gerkensmeyer JE, Joyce BA, et al. Reliability and validity of the faces and word descriptor scales to measure procedural pain. J Pediatr Nurs 1996;11:368-74.

115. Robertson J. Pediatric pain assessment: validation of a multidimensional tool. Pediatr Nurs 1993;19:209-13.

116. Stein PR. Indices of pain intensity: construct validity among preschoolers. Pediatr Nurs 1995;21:119-23.

117. West N, Oakes L, Hinds PS, et al. Measuring pain in pediatric oncology ICU patients. J Pediatr Oncol Nurs 1994;11:64-8. discussion 69-70.

118. Bond FW, Hayes SC, Baer RA, et al. Preliminary psychometric properties of the acceptance and action questionnaire-II: a revised measure of psychological inflexibility and experiential avoidance. Behav Ther 2011;42:676-88.

119. Brown TA, Chorpita BF, Korotitsch W, et al. Psychometric properties of the Depression Anxiety Stress Scales (DASS) in clinical samples. Behav Res Ther 1997;35:79-89.

120. Driscoll K, Pianta RC. Mothers' and fathers' perceptions of conflict and closeness in parent-child relationships during early childhood. JECIP 2011;7:1.

121. Pianta RC, Steinberg M. Teacher-child relationships and the process of adjusting to school. New Dir Child Adolesc Dev 1992;1992:61-80.

122. Kendall PC, Sheldrick RC. Normative data for normative comparisons. J Consult Clin Psychol 2000;68:767-73.

123. Lehr R. Sixteen S-squared over D-squared: a relation for crude sample size estimates. Stat Med 1992:11:1099-102.

124. Braun V, Clarke V. Using thematic analysis in psychology. Qual Res Psychol 2006;3:77-101. 